\title{
Collet-Sicard syndrome as an initial presentation of prostate cancer: a case report
}

Rosa Villatoro ${ }^{1 *}$, Carlos Romero ${ }^{2}$ and Antonio Rueda ${ }^{1}$

\begin{abstract}
Background: Collet-Sicard syndrome is caused by lesions at the base of the skull affecting the lower cranial nerves. It is associated with various etiologies of tumoral and other origin. Although this syndrome has been reported previously in the literature, most cases are diagnosed as part of primary disease follow-up. This case is unusual because of the diagnosis of bone metastasis secondary to prostate cancer.

Case presentation: We present the case of a 70-year-old Caucasian man with a three-week history of headache and maxillary pain on the right side together with paresis of the low cranial nerves. This study was carried out with a computed tomography (CT) scan of the larynx and neck and MRI, which revealed a bone lesion at the base of the skull affecting the right occipital condyle and part of the right side of the basilar bone. On the basis of differential diagnosis, a fibrous dysplasia, Paget's disease or metastasis was considered. Finally, and after other studies were performed, a diagnosis of bone metastasis secondary to prostate cancer was established.

Conclusions: We think that this case is curious because it involved an initial presentation of metastatic prostate cancer. It is important this should be considered in the differential diagnosis when a patient with unusual clinical findings is first seen in view of the fact that first-line hormonal treatment may control the disease for months or years.
\end{abstract}

\section{Background}

Collet-Sicard syndrome is caused by lesions at the base of the skull affecting the lower cranial nerves, which produces dysphonia, displacement of the palate, and atony of the trapezius muscle and sternocleidomastoid, as well as anesthesia of the larynx, pharynx and soft palate. It is associated with various etiologies of tumoral and other origins. The differential diagnosis is important. Among the nontumoral factors causing Collet-Sicard syndrome, the most common are traumatic events (fractures at the base of the skull, aneurisms, and so on), inflammatory processes (osteomyelitis, Paget's disease, and so on) or other alterations such as diabetes mellitus or porphyrias [1]. However, considering a potential tumor cause in the differential diagnosis is important.

Collet-Sicard syndrome may be diagnosed based on clinical history, a physical examination or imaging studies such as computed tomography (CT) and MRI scans [1].

\footnotetext{
* Correspondence: villatororoldan@gmail.com

'Unidad Oncologia Médica, Autovia A-7, km 187, Hospital Costa del Sol, Marbella, 29603, Spain

Full list of author information is available at the end of the article
}

The site most frequently affected is the petrous apex, although the external auditory canal, the middle ear and the mastoid apophysis can also be involved [2]. The symptoms vary depending on the location of occurrence, producing effects ranging from loss of hearing to tinnitus or disorders of cranial nerve VIII, the jugular foramen or the anterior condylar canal. The latter is the site described in our patient's case [3].

\section{Case presentation}

We report the case of a 70-year-old Caucasian man, with no significant clinical background, who presented to our casualty department with a three-week history of headache and maxillary pain on the right side, together with the recent appearance of dysphonia and dysphagia for solids. No urinary disorder was reported. A neurological examination revealed a paresis of cranial nerves IX and $\mathrm{X}$, manifested by the displacement of the soft palate to the right and difficulty swallowing. Paresis of cranial nerves XI and XII was also observed, indicated by the lowering of the right shoulder and hypotonia of the right trapezius muscle, and was accompanied by displacement 
of the tongue toward the right, right-side hypotonia and muscle twitching (Figures 1 and 2). Results of the rest of the physical examination were normal.

Blood analysis results revealed an alkaline phosphatase level of $350 \mathrm{UI} / \mathrm{L}$ (normal range 44 to $147 \mathrm{UI} / \mathrm{L}$ ) but no other significant alterations. In view of the paresis of the four lower cranial nerves, a CT scan of the larynx and neck was performed; the CT scan revealed an asymmetrical union between the clivus and the right occipital condyle, adjacent to the jugular foramen, with increased ground-glass bone density. There was no visible lesion to the bone cortex or soft tissues. This study was complemented with an MRI scan, which revealed a bone lesion producing a hypointense signal at sequences $\mathrm{T} 1$ and $\mathrm{T} 2$. Administration of a gadolinium contrast agent produced a moderate degree of enhancement at the base of the skull, affecting the right occipital condyle and part of the right side of the basilar bone (Figure 3). The image corresponded to a moderately space-occupying blastic lesion, visible in the CT scan, which slightly decreased the caliber of the jugular foramen and the condylar canal. Therefore, fibrous dysplasia, Paget's disease and metastasis were considered in the differential diagnosis.

Subsequently, an additional radiographic examination of the lumbar column and pelvis was carried out; this examination did not reveal any lesions suggestive of Paget's disease. A bone gammagraphy was then requested, and images showed multiple pathological foci of tracer uptake in the right maxilla, the rib cage, right scapula, spine and pelvis. These foci were compatible with disseminated bone metastases. The blood analysis was repeated, and tumor markers were studied. The initial prostate-specific antigen (PSA) value was $21.30 \mathrm{ng} / \mathrm{mL}$.

A physical examination revealed an enlarged prostate with a hard consistency, destructured in the left lobe. Because there was a strong suspicion of prostate

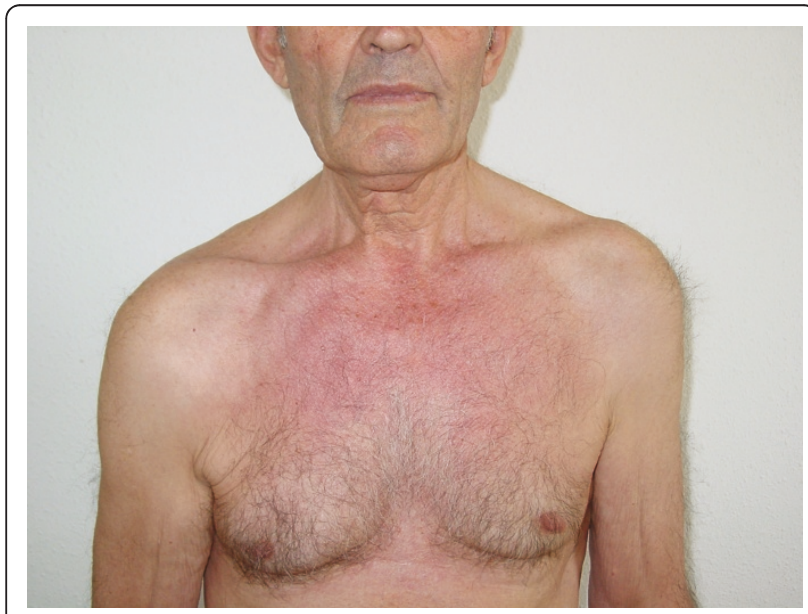

Figure 1 Paresis of cranial nerve $\mathrm{XI}$

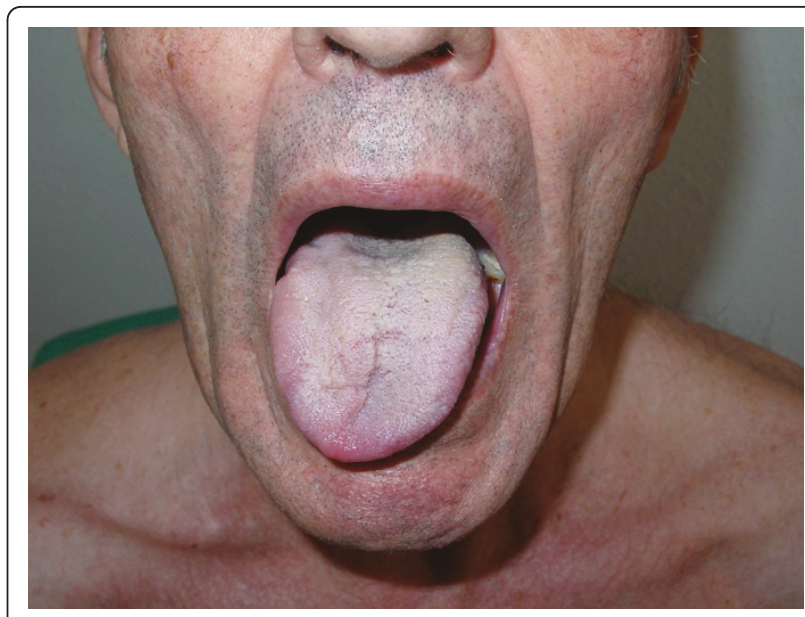

Figure 2 Paresis of cranial nerve XII.

neoplasm, a biopsy was performed. The anatomic pathology findings were bilateral common adenocarcinoma, with a Gleason grade of $8(4+4)$, affecting $60 \%$ of the tissue. There was no presence in the periprostatic adipose tissue and no perineural infiltration.

Following the diagnosis of stage IV prostate adenocarcinoma by metastatic bone dissemination with ColletSicard or jugular foramen syndrome, hormone treatment was begun with an antiandrogen. Then, 15 days later, a luteinizing hormone-releasing hormone (LHRH) analog

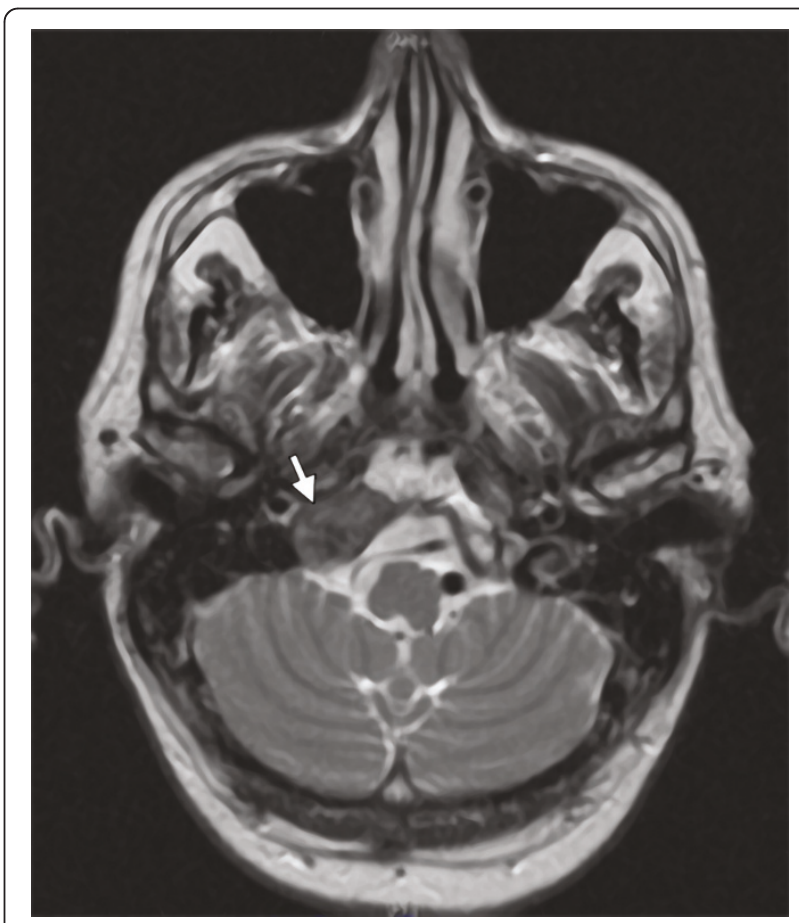

Figure 3 MRI scan with moderate degree of enhancement at the base of the skull, affecting the right occipital condyle and part of the right side of the basilar bone. 
was added and a monthly dose of zoledronic acid was subsequently included. The PSA value during the diagnostic process, prior to the start of antiandrogen therapy, was $71.9 \mathrm{ng} / \mathrm{mL}$.

After three months of treatment, our patient was able to swallow normally, but the dysphonia remained. The rightward displacement of the palate, the lowering of the right shoulder and the atony of the right side of the tongue (paresis of cranial nerves XI and XII) remained unaltered. The latest PSA value was $0.11 \mathrm{ng} / \mathrm{mL}$.

\section{Discussion}

The clinical presentation of metastasis to the temporal bone is uncommon, and few cases have been reported. Nevertheless, its incidence is probably greater than commonly estimated because of the number of cases that remain undiagnosed. The multi-symptom nature of metastatic bone disease tends to produce more incapacitating symptoms than those associated with diseases of the temporal bone.

Various retrospective series of patients presenting with this syndrome have been reported in the literature. Vázquez et al. described 21 cases, of which $71 \%$ were secondary to neoplasia (57\% from paraganglioma and $14 \%$ by the direct extension of carcinoma of the cavum) [1]. Imamura et al. reviewed the potential mechanisms responsible for metastatic dissemination to the temporal bone. Of the six patients studied, three cases presented hematogenous dissemination (hepatocellular carcinoma, non-microcytic lung cancer and adenocarcinoma of unknown origin), two cases were the consequence of direct invasion by carcinoma of the head and neck, and one case was caused by leptomeningeal carcinomatosis (carcinoma of transitional cell carcinoma of the renal pelvis) [4]. Gloria-Cruz et al. selected 212 corpses of patients with non-disseminated neoplasias for an autopsical study of the temporal bone. These authors identified 47 patients with metastasis in the temporal bone, and the involvement was bilateral in $62 \%$ of these cases. The most frequently occurring site was the petrous apex, and the hematogenous pathway was the normal route of dissemination $[5,6]$.

The management of Collet-Sicard syndrome consists of treating the cause that originates. In this case, therapy over primary tumor, followed by other measures such as the use of steroids or radiotherapy to help reduce edema and, thus, alleviate symptoms that can be limiting for the patient [7].

\section{Conclusions}

The medical literature contains various descriptions of patients with disseminated prostate cancer who presented with Collet-Sicard syndrome; however, in almost every case, this diagnosis was already known when neurological symptoms began [7-12]. Apart from our patient, only one other case has been reported where metastasis to the temporal bone was the first recognized symptom of the disease [12]. It is important to consider the possibility of the existence of prostate cancer when a patient with an unusual clinical presentation is first seen, in view of the fact that first-line hormonal treatment may control the disease for months or years.

\section{Consent}

Written informed consent was obtained from the patient for publication of this case report and any accompanying images. A copy of the written consent is available for review by the Editor-in-Chief of this journal.

\section{Author details}

'Unidad Oncologia Médica, Autovia A-7, km 187, Hospital Costa del Sol, Marbella, 29603, Spain. ²Servicio Medicina Interna, Autovia A-7, Km 187, Hospital Costa del Sol, Marbella, 29603, Spain.

\section{Authors' contributions}

CR made substantial contributions to the design, and the acquisition and interpretation of data. AR revised the manuscript critically for important intellectual content. RV was a major contributor in writing the manuscript. All authors read and approved the final manuscript.

\section{Competing interests}

The authors declare that they have no competing interests.

Received: 7 October 2010 Accepted: 14 July 2011

Published: 14 July 2011

\section{References}

1. Vázquez BV, Saynes MFJ, Hernández VG: Sindrome de Agujero Rasgado posterior. Casuística y manejo. An Orl Mex 2002, 47:4-8.

2. Belal A Jr: Metastatic tumours of the temporal bone. A histopathological report. J Laryngol Otol 1985, 99:839-846.

3. Syms MJ, Singson MT, Burgess LP: Evaluation of lower cranial nerve deficits. Otolaryngol Clin North Am 1997, 30:489-463.

4. Imamura S, Murakami Y: Secondary malignant tumor of the temporal bone. A histopathologic study and review of the world literature. Nippon Jibiinkoka Gakkai Kaiho 1991, 94:924-937.

5. Gloria-Cruz TI, Schachern PA, Paparella MM, Adams GL, Fulton SE: Metastases to temporal bones from primary nonsystemic malignant neoplasms. Arch Otolaryngol Head Neck Surg 2000, 126:209-214.

6. Nelson EG, Hinojosa R: Histopathology of metastatic temporal bone tumors. Arch Otolaryngol Head Neck Surg 1991, 117:189-193.

7. Chacon G, Alexandraki I, Palacio C: Collet-Sicard syndrome: an uncommon manifestation of metastatic prostate cancer. South Med J 2006, 99:898-899.

8. Shine NP, O'Sullivan P: Collet-Sicard syndrome: a rare presentation of metastatic prostate adenocarcinoma. Auris Naus Larynx 2005, 32:315-318.

9. Prashant R, Franks A: Collet-Sicard syndrome-a report and review. Lancet Oncol 2003, 4:376-377.

10. Satoh H, Nishiyama T, Horiguchi A, Nakashima J, Saito S, Murai M: A case of Collet-Sicard syndrome caused by skull base metastasis of prostate carcinoma. Nippon Hinyokika Gakkai Zasshi 2000, 91:562-564.

11. Wilson $\mathrm{H}$, Johnson $\mathrm{DH}$ : Jugular foramen syndrome as a complication of metastatic cancer of the prostate. South Med J 1984, 77:92-93.

12. Onishi A, Shida K: Case of Collet-Sicard syndrome due to metastasis of prostatic cancer. Naika 1970, 26:755-757.

doi:10.1186/1752-1947-5-315

Cite this article as: Villatoro et al:: Collet-Sicard syndrome as an initial presentation of prostate cancer: a case report. Journal of Medical Case Reports 2011 5:315. 